\title{
Fortifications and landscape system: geological and geomorphological resilience in the development of the La Spezia Gulf
}

\author{
Serena di Grazia ${ }^{a}$, Ludovica Marinaro ${ }^{b}$
}

${ }^{\mathrm{a}}$ Geologist, Florence, Italy, geol.digrazia@gmail.com, ${ }^{\mathrm{b}}$ Dipartimento di Architettura, Florence University, Italy, ludovica.marinaro@unifi.it

\begin{abstract}
The gulf of La Spezia, in the North-Western part of the Mediterranean coast, harbours in its profound arc a rich and complex system of fortifications, which finds in the Maritime Military Arsenal (1869) its propelling force. Napoleon was amongst the first to build a key military base in this strategic location which was a fortification in itself. This study uses landscape structure analysis, geomorphological studies and cartographic recognitions to highlight how a different approach to the landscape management affects the resilience of the site, especially while accomplishing military objectives. This trans-disciplinary process will highlight how historical and social changes relate to different approaches in land management; in particular how political objectives (such as defence and control of territory) reflect a precise idea of landscape. The final aim will be to identify key features that allow an accurate re-reading of the landscape itself.
\end{abstract}

Keywords: Landscape resilience, Geology, geomorphological analysis, Trans-disciplinarity.

\section{Introduzione}

Recuperare il concorso delle discipline di indagine scientifica del territorio quali la geologia, la geomorfologia e l'idrologia nel processo di interpretazione del paesaggio, significa comprendere che tutti gli usi antropici, dall'abitare sino alle singole specializzazioni funzionali, interagiscono con una realtà a tre dimensioni. La profondità del suolo e lo strato aereo sono quindi parte integrante del territorio e partecipano alla sua continua trasformazione, ma i tentativi di rigenerazione territoriale attuati mediante i piani urbanistici si concentrano ancora su una dimensione puramente estensiva, sono piani di superficie che procedono per lotti.

Nello specifico caso del golfo della Spezia il primo esempio di questo modus operandi si ha a partire dal 1860 c.a. con i progetti per l'Arsenale Marittimo Militare, opera che segna un netto cambio di approccio alla pianificazione territoriale nel golfo lasciando chiara impronta nell'attuale cultura progettuale. La costruzione dell'arsenale ha segnato una frattura nel ciclico e spontaneo tramutare del golfo da località balneare a porto militare, un segno pesante che ha modificato la struttura geomorfologica e idrogeologica del territorio, generando una struttura urbana dominata dalla griglia ottocentesca della città umbertina in cui $\mathrm{i}$ torrenti, l'orografia del colle dei Cappuccini, la famosa "polla di Cadimare" e tutte le originali caratteristiche naturali vengono con ostinazione cancellate l'una dopo l'altra, prima dalla cartografia e poi dal territorio e dalla memoria della popolazione, condizionando in modo decisivo la percezione del paesaggio e il suo ricco immaginario. 


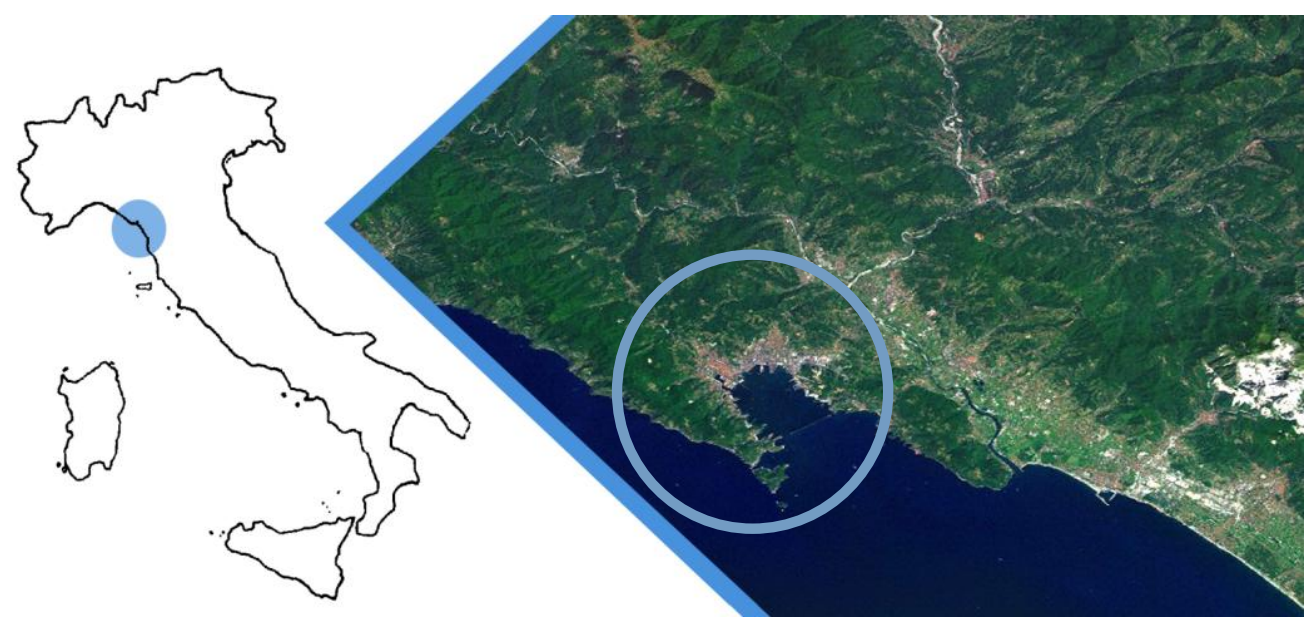

Fig. 1- Inquadramento del golfo della Spezia, arco latino del Mediterraneo occidentale

Lo studio delle dinamiche che hanno concorso alla creazione del paesaggio fortificato del Golfo della Spezia, recupera la "profondità" mettendo in luce le relazioni che intercorrono tra la geologia, la naturale vocazione del territorio e la sua intrinseca capacità di rigenerarsi e sostenersi nel tempo. Ciò permette altresì di comprendere come il patrimonio architettonico delle fortificazioni, inteso nell'unità sistemica del golfo, sia oggi in grado o meno di confermare il "ruolo" che gli è stato conferito con il progetto dell'arsenale e di dare a tale ruolo nuovi significati nel processo di trasformazione del paesaggio. Le architetture militari disseminate su tutto l'arco costiero sono un elemento caratterizzante del paesaggio della Spezia. Oggi che la base militare ha perso progressivamente importanza in favore di quella di Taranto nel cuore del Mediterraneo, tali strutture sono in disuso e si avanza verso una progressiva dismissione.

Proseguendo nella politica territoriale prima descritta, che di fatto parcellizza il territorio, si corre il rischio di compromettere i caratteri distintivi di tale complesso, il cui pregio risiede nell'unitarietà del disegno del sistema di fortificazioni a scala paesaggistica. Disegno che, seppur con grande impatto, riflette una precisa idea di territorio in cui opera architettonica e geomorfologia concorrevano a dare corpo all'immagine del golfo militare della Spezia.
Tutti gli interventi di restauro e riconversione del patrimonio architettonico delle fortificazioni devono perciò prendere in considerazione prioritariamente le relazioni che intercorrono tra architetture e paesaggio grazie ad un approccio transdisciplinare che deve progressivamente coinvolgere le discipline atte a restituire una capacità di lettura delle caratteristiche del territorio.

L'analisi geomorfologica contribuisce a raggiungere un duplice obiettivo: da un lato rifondare un approccio progettuale multidisciplinare e transcalare attento alle dinamiche naturali del territorio; dall'altro, l'identificazione dei motivi strutturali e dei loro processi evolutivi come elementi costituenti l'identità naturale del luogo, permette di valutare le potenzialità e i limiti del paesaggio del golfo della Spezia e di individuare la capacità resiliente residua di tali risorse, elementi che vanno a costituire la base documentativa essenziale per proporre un progetto di riqualificazione del patrimonio delle fortificazioni militari.

\section{Analisi geomorfologica}

Come un carattere, il territorio è il risultato dell'evoluzione delle diverse matrici di cui è costituito. Il processo di analisi prevede il recupero delle informazioni geologiche ed il 
riconoscimento delle caratteristiche peculiari dell'area. Per comprendere la natura e il naturale sviluppo di tali elementi si è provveduto a descriverne la genesi e l'evoluzione nel tempo.

Ad oriente ed occidente il golfo è chiuso da due dorsali parallele orientate NO-SE, si riporta in figura 2 lo schema strutturale in cui si distinguono le diverse unità geologiche.

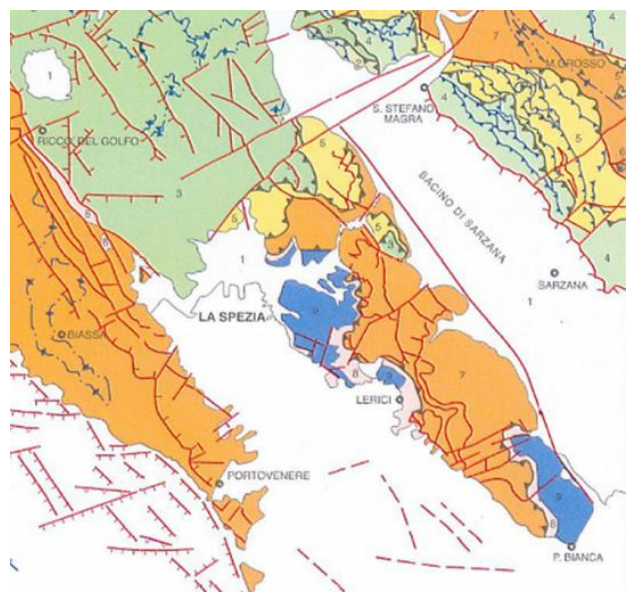

Fig. 2- Schema strutturale (Abbate E., 2005).

La dorsale di occidente è una piega anticlinale rovesciata con vergenza tirrenica (Fig. 3). Il fianco verso il mare aperto è costituito dalle arenarie della Formazione del Macigno disposte in strati sub-perpendicolari (formazione MAC Fig. 3), mentre lungo il versante interno al golfo affiorano i membri calcarei della Falda Toscana (formazioni da MAI a LSP, Fig. 3). Le rocce calcaree sono sede di un complesso sistema carsico ipogeo che, seguendo l'assetto strutturale degli strati e le linee di frattura presenti, convoglia le acque meteoriche all'interno del golfo. La dorsale orientale, per la sua articolata struttura geologica è caratterizzata da una maggiore irregolarità nelle forme e nell'idrografia. Affiorano arenarie e quarziti, le rocce carbonatiche sono limitate ai rilievi a SE nelle località di Fontanaviva e Pietralba.

La struttura geologica dell'area è legata all'orogenesi appenninica, fase compressiva in cui sedimenti depositati nelle profondità di un braccio del paleo-oceano Tetide sono stati portati in superficie, piegati ed impilati in falde.
A fasi compressive sono succedute fasi distensive dove faglie dirette, tra cui quella della Spezia che taglia il golfo con direzione NO-SE, hanno creato una serie di depressioni parallele quali il golfo di Spezia e poco a Sud il bacino di Sarzana (Fig. 2).

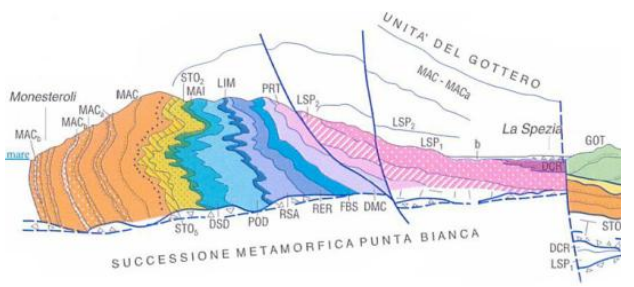

Fig. 3 - Sezione geologica del promontorio occidentale (Note illustrative Carta Geologica d'Italia, 2005)

A tali movimenti tettonici ne sono seguiti altri durante i quali si ebbe il sollevamento delle colline retrostanti il golfo. Questi movimenti tra la fine del Pleistocene e l'inizio dell'Olocene coincisero con la fine della glaciazione wurmiana e provocarono notevoli cambiamenti nell'assetto idrografico: il fiume Vara che fino ad allora sfociava nel golfo di Spezia (al tempo la linea di costa era arretrata rispetto all'attuale) con l'innalzamento dell'area e la formazione delle colline, ha modificato il suo corso confluendo nel fiume Magra che sfocia poco a Sud nel bacino di Sarzana.

Durante l'Olocene il livello del mare risalì fino a riempire i due bacini, così mentre quello di Sarzana veniva lentamente colmato dai sedimenti del Magra, quello di Spezia, non più soggetto a dinamiche fluviali importanti, sostanzialmente mantenne le stesse profondità. Confrontando il bacino di Spezia con quello di Sarzana è interessante vedere come, partendo da una stessa struttura, ad una diversa evoluzione del territorio sia connesso uno specifico sviluppo urbano ed economico. Se nel bacino di Sarzana il fiume ha garantito una via utile per il commercio permettendo la fioritura della civiltà mercantile di Luni, (I secolo a.C.), il golfo di Spezia, a seguito della deviazione del Vara, ha mantenuto una profondità del fondale marino tale da confermare la sua vocazione portuale, garantendo l'accesso a navi sempre più grandi. A queste considerazioni si aggiunge che le 
ancora "giovani" colline retrostanti il golfo costituivano una barriera per gli scambi commerciali, ma allo stesso tempo offrivano un facile controllo delle vie d'accesso, condizione essenziale per un sito militare.

Il golfo attualmente è interessato da una serie di canali e torrenti di cui i principali, sono il Lagora ad ovest ed il torrente Cappellini ad Est (Fig. 4), entrambi con un modesto bacino di raccolta. L'asimmetria nella struttura geologica dei promontori comporta differenze nella circolazione idrica: se in quello orientale prevale il deflusso superficiale, per il promontorio ad ovest giocano un ruolo fondamentale i fenomeni carsici delle formazioni calcaree che danno luogo ad una circolazione ipogea. L'acqua piovana si infiltra nelle cavità carsiche del versante per riemergere in pressione (circa 5 bar) a valle, sia nei depositi di spiaggia su cui è stata costruita la città, che direttamente in mare. Questo fenomeno ha creato "polle" e tipici laghetti detti "sprugole". Le "sprugole" conosciute e sfruttate fin dall'antichità, variavano notevolmente le dimensioni a seconda dell'apporto idrico e venivano utilizzate sia per l'approvvigionamento che per il funzionamento di mulini e frantoi. Risalite di acque minerali sono state documentate anche nel promontorio orientale, negli studi condotti dagli ingegneri Le Père (1810 ca.) e Peyron (1846 ca.), vicino all'abitato di Pitelli e poco a nord della batteria di S.Bartolomeo.

Con l'analisi geomorfologica delle aree sommerse si comprende il legame che intercorre tra dinamiche pelagiche e le aree prossime al litorale rilevandone i principali fattori generatori. Indagini acustiche effettuate all'interno del golfo in occasione della redazione della Carta Geologica d'Italia (progetto CARG) hanno rilevato i corpi sedimentari che costituiscono il fondale del golfo.

La presenza della foce del fiume Vara all'interno del golfo fino al Pleistocene ha determinato la deposizione di corpi sedimentari progradanti solcati da canali. Dopo la cattura del Vara nell'Olocene diminuisce drasticamente il tasso di sedimentazione e si crea alla bocca del golfo una barra sabbiosa trasversale che ha permesso l'instaurarsi di condizioni lagunari nella porzione orientale del golfo (zona di Patch-Reef in figura 4).

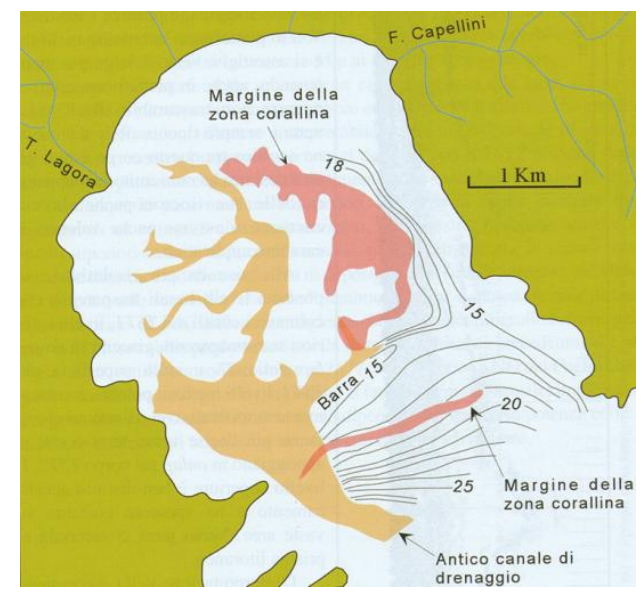

Fig. 4 - Schema della paleogeografia della parte interna del golfo durante l'inizio dell'Olocene (Abbate E., 2005).

E' da notare come le paleomorfologie ricalcano l'attuale condizione del fondale del golfo, in particolare il canale ha mantenuto la stessa posizione durante i millenni. Dal'inizio del'Olocene quindi il golfo nella sua metà occidentale è caratterizzato da canali mentre nella metà orientale è presente una sedimentazione di mare basso e lagunare. La stessa morfologia si è mantenuta nel tempo fino all'attuale con l'area degli "stagnoni" nella metà orientale del golfo ed il canale parallelo al promontorio occidentale, che ha mantenuto la sua posizione pressoché invariata nonostante abbia subito numerosi rimaneggiamenti nell'ultimo secolo.

\section{Trasformazione del paesaggio}

$\mathrm{Al}$ porto della Spezia e non ancora alla città fanno riferimento le prime voci su quello che sarebbe diventato il golfo dei poeti, testimonianze che ne accertano la colonizzazione romana proprio in virtù della sua posizione strategica nel Mediterraneo. Da sempre il golfo mantenne un'importanza militare cui si alternò nel corso della storia soltanto la fama di località di bagni e vacanza, come testimoniano le ville romane del Muggiano e del 
Varignano. Fu però a cavallo del secolo XIX, che la trasformazione del paesaggio del Golfo venne indirizzata in modo quasi irreversibile verso il ruolo di base militare strategica, fatto che determinò anche la trasformazione del patrimonio architettonico delle fortificazioni. Su questo momento cruciale si concentra l'analisi della cartografia che oltre a dare indicazioni preziose sullo stato dei luoghi, comunica come veniva percepita ed interpretata la qualità del paesaggio. Nella carta di figura 5, G.Brusco e G.Ferretto rappresentano l'area oggi occupata dall'arsenale, cuore dell'antica piana agricola di San Vito, riportando fedelmente il sistema dei corsi d'acqua per dare una precisa gerarchia ai lavori di regimentazione idraulica fatti e da farsi. L'andamento sinuoso della linea di costa non è condizionato da opere antropiche ma dal solo sbocco del torrente Lagora e di tutti i torrenti minori che innervano la piana insieme alla "sprugola" al limite ovest dell'antica cittadella di Spezia. La viabilità antica correva parallela al litorale e ai corsi d'acqua cadenzando il ritmo dei poderi agricoli. In questa carta si ha la sensazione di un paesaggio in attesa: si può cogliere la tensione della città antica che entro le mura racchiude ancora campi ma freme per conquistare la linea di battigia. Gli studi e i lavori per la sistemazione idrogeologica della piana, così puntuali e meticolosi sul Lagora e sui torrenti al confine ovest della città rivelano il desiderio di dominio e fanno pensare a un progetto più ambizioso rispetto alla semplice espansione urbana, che per altro senza una ragione economica e politica non sussisterebbe.

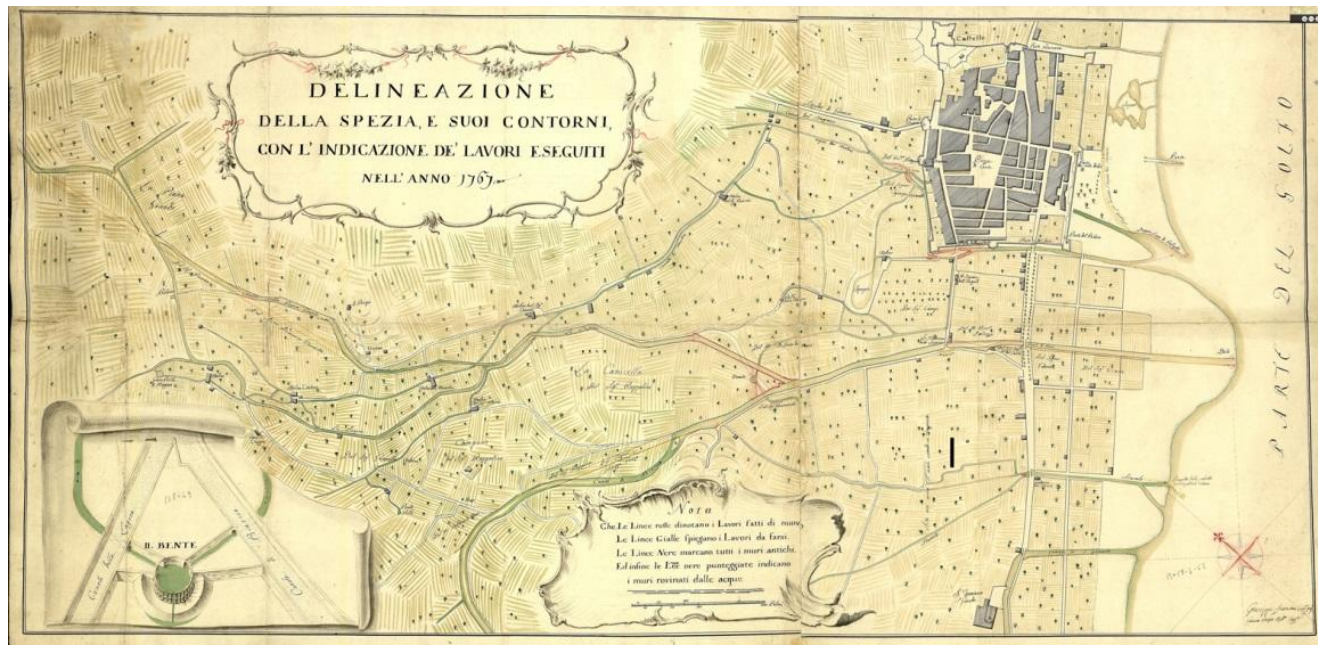

Fig. 5- Carta del golfo della Spezia (G. Brusco e G.Ferretto, 1767)

Napoleone arrivò a Spezia nel 1800 e con decreto del 11 maggio 1808 dichiarò il golfo della Spezia porto militare. Nel 1810 diede incarico al capo di battaglione P.A. Clerc di avviare un'imponente campagna di rilievo, per determinare il sito idoneo alla costruzione di quello che, secondo le sue ambizioni, doveva essere "il più grande stabilimento marittimo dell'universo" (IGM, 2005). La carta i figura 6 riflette appieno la dicotomia secondo cui veniva immaginato il futuro del golfo, tra l'immagine di locus amoenus dall'inestimabile pregio naturalistico e la vocazione militare che il genio napoleónico vedeva altrettanto naturale. Al Clerc si debe il rilievo di tutta la costa occidentale del golfo con la prima applicazione sistematica del metodo delle curve di livello e una serie di bellissime vedute ad acquerello.

Il nuovo metodo conferisce ancora maggiore risalto alla complessa storia geomorfologica del territorio, a cui la rappresentazione cartografica dà particolare rilevo fondendo insieme arte $\mathrm{e}$ necessità per testimoniare l'armonia di un golfo non ancora contaminato. Tale rappresentazione era funzionale a comprendere il comportamento 
del territorio per poter calibrare l'inserimento della base militare in modo coerente alle caratteristiche del luogo.

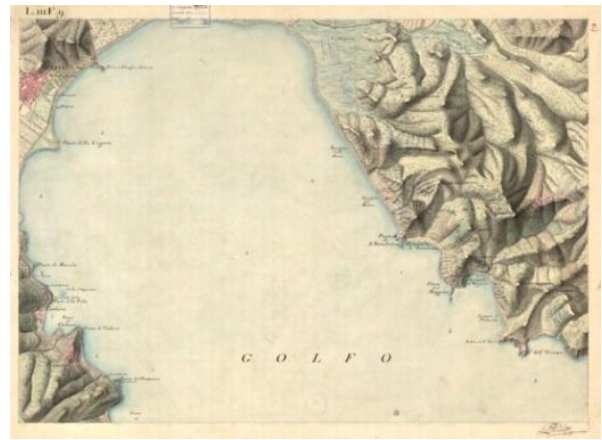

Fig.6 - Carta (Pierre Antoine Clerc, 1810)

Dai progetti napoleonici, rimasti solo su carta ma che hanno profondamente condizionato l'immaginario collettivo, si passa nel 1860-61 a quelli definitivi del generale D.Chiodo (Fig. 7). L'arsenale si impone sulla piana di San Vito con un'estensione di 165,49 ha, cui si aggiungono i cantieri navali di San Bartolomeo sulla costa orientale per una superficie totale di 179,31 ettari, che fanno dell'opera di Chiodo il più grande intervento militare Europeo dell'Ottocento. L'opera trasfigura completamente la geomorfologia dei luoghi dall'entroterra al litorale, con forte incidenza sull'idrogeologia originaria, conferma la visione programmatica del golfo come "macchina militare" in un disegno unitario, e si pone come "fattore di rottura spazio-temporale", compromettendo cioè la capacità del territorio di alternare secondo le necessità dei tempi, il ruolo di porto militare a quello di località balneare. Scompaiono dalla carta tutti i riferimenti agli elementi naturali, sistematicamente cancellati poiché il territorio viene concepito come semplice substrato per l'arsenale che assume una struttura spaziale di tipo urbanistico, ampiamente fuori scala rispetto alle dimensioni degli insediamenti nel golfo ed al sistema di relazioni, tanto da fondare una nuova gerarchia funzionale. Ciò ha determinato un forte disequilibrio dell'intera geomorfologia del contesto limitandone la percezione e l'uso, condizionando lo sviluppo della città e di tutto il territorio circostante.

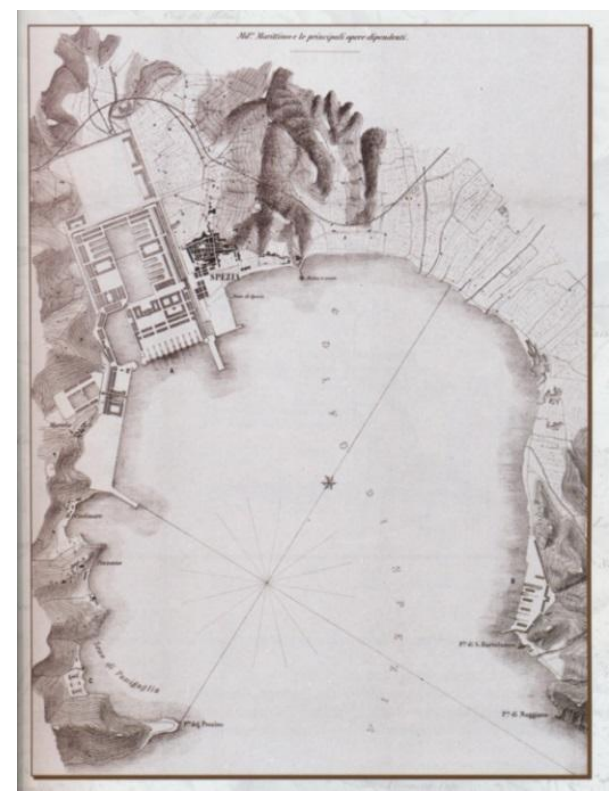

Fig. 7- Pianta dell'arsenale della Spezia (Domenico Chiodo, 1863)

\section{Considerazioni sulla resilienza}

Lo studio della resilienza degli elementi caratterizzanti diventa un utile strumento per un nuovo approccio al progetto di riconversione e riqualificazione dei luoghi, approccio orientato alla sostenibilità sia in chiave ecologico ambientale che sociale.

Si definisce resilienza la velocità, la capacità di un sistema di ritornare allo stato iniziale, in questa sede si prende in considerazione il sistema geomorfologico del territorio rapportandoci alle fasi progettuali che si sono succedute nell'area. Il golfo è caratterizzato dai rilievi, intesi come promontori e sistema collinare, dal fenomeno delle "sprugole", dalla piana costiera fortemente urbanizzata e comprendente l'area degli "stagnoni", e dal reticolo di canali e fossi regimati e quasi completamente tombati.

\subsection{Morfologia dei rilievi}

L'attività estrattiva nell'area è documentata fin dal II secolo a.C., sono noti i marmi di "Punta Bianca", estremità a mare del promontorio orientale e anche le cave sia a cielo aperto che 
sotterranee per l'estrazione del calcare "Portoro" nel promontorio occidentale, mentre cave di arenaria aperte negli strati massivi del Macigno erano utilizzate per la costruzione e la pavimentazione delle strade dal 1900. All'estrazione della pietra come risorsa lapidea si aggiungono gli scavi attuati a seguito della costruzione dell'Arsenale con la creazione delle darsene, dei bacini e di volumi ipogei nel monte "Parodi" come depositi e rifugi bellici, opere delle quali non si ha documentazione in quanto vincolati da segreto militare. La condizione naturale preesistente è stata modificata in una forma definitiva. L'utilizzo delle gallerie allinterno della montagna per lo stoccaggio di materiali inoltre ha interferito con il particolare assetto idrogeologico ed ha comportato un forte inquinamento delle matrici ambientali. La progettazione urbanistica successiva alla realizzazione dell'arsenale, dal 1900 circa è stata anch'essa particolarmente incisiva e con la demolizione del colle dei Cappuccini, rilievo al centro del golfo che separava l'area degli "stagnoni" dall'abitato di Spezia, e dell'isolotto antistante ha modificato in maniera irreversibile la morfologia del golfo. La resilienza in questo contesto è nulla, il progetto di riqualificazione dell'area potrà comunque cercare di ristabilire un legame percettivo con quello che è il carattere naturale del luogo.

\subsection{Idrologia}

L'intervento antropico sulla componente idrologica del territorio è stato sicuramente tra $\mathrm{i}$ più incisivi così da condizionare e stravolgere nel corso dell'ultimo secolo la percezione del paesaggio di un intera porzione del golfo, come è evidente dai numerosi dipinti e dalle raffigurazioni ottocentesche. L'antica zona palustre è stata bonificata e la maggior parte dei canali è stata tombata secondo un programma di controllo che contrasta il naturale sviluppo idrografico dell'area. Il fenomeno delle "sprugole" è stato cancellato dal territorio e dalla cartografia del XX secolo, ma da quanto si evince dalla struttura geologica risulta chiaro che si tratta di un fenomeno rinnovabile perenne, legato alle piogge ed è una fonte inesauribile di acqua dolce.
La geomorfologia sommersa ha mantenuto negli anni la stessa fisiografía mostrando un'alta resilienza. Dal Pleistocene ad oggi la posizione del canale e dell'area paludosa sono rimaste sostanzialmente invariate nonostante gli interventi di bonifica e le modifiche dovute all'attuazione dei progetti militari.

Nonostante il forte impatto determinato dalla costruzione dell'Arsenale, le cui opere hanno interferito con la circolazione ipogea, la resilienza del sistema idrologico è alta.

\section{Conclusioni}

È molto raro che ci si possa confrontare con la dismissione di complessi dell'entità della base militare di Spezia in tutta la sua articolazione territoriale. Si tratta di occasioni irripetibili per proporre una nuova idea di paesaggio, necessaria in contesti territoriali fortemente urbanizzati, caratterizzati da tessuti stratificati e sovrascritti spesso senza una grammatica durante e dopo il boom economico. Oggi che siamo alle porte di una necessaria transizione energetica verso fonti rinnovabili, l'idea di paesaggio non è e non può essere disgiunta da una nuova idea di sfruttamento delle risorse del territorio. La ricostruzione geologica e idrologica messa a sistema con l'analisi paesaggistica dell'area permette di individuare gli elementi strutturali che caratterizzano il paesaggio in forma più o meno percepibile, con l'intento di formare una "conoscenza adeguata" del territorio (Pascucci, 2006) e non di cristallizzarli in vincoli. Infatti i caratteri idro-geo-morfologici ed i sistemi morfogenetici, che sono considerati vere e proprie invarianti strutturali nei più recenti strumenti urbanistici, al di là della previsione del sistema vincolistico che ne preservi l'assetto non vengono messi a sistema nei progetti come elementi concorrenti al naturale e spontaneo "funzionamento" del territorio, con l'effetto conseguente di trascurare il loro ruolo nella trasformazione antropica del paesaggio, di perdere la capacità di saper progettare con essi e soprattutto di saperli gestire, aspetto non trascurabile alla luce dei recenti dissesti idrogeologici. Ad oggi la mancata applicazione 
dei principi della Convenzione Europea del Paesaggio (CEP, 2000, Firenze) rende difficile attuare quella rivoluzione culturale necessaria a proporre una nuova idea di paesaggio. Il progetto, necessariamente transdisciplinare, è il solo strumento efficace di cui possiamo disporre. Pensare ad un progetto di riqualificazione e riconversione del sistema di fortificazioni del golfo di Spezia, significa pertanto innescare un processo: quella "Ricercazione" (Pizziolo, 2003) che questo studio si propone di alimentare, integrando una dettagliata indagine scientifica, l'esperienza diretta sul territorio e il dialogo con la cittadinanza in un ciclo continuo, in un processo che mira a recuperare l'unitaria del territorio per saper cogliere il senso dei luoghi ancor prima che promuovere degli interventi puntuali sul patrimonio architettonico storico.

\section{Note}

Si omettono le legende delle carte geologiche e strutturali perché non influenti per la comprensione degli argomenti trattati. Tali carte hanno la funzione di chiarire visivamente l'assetto strutturale degli elementi geologici e geomorfologici. Per ulteriori dettagli ed approfondimenti si rimanda alle opere citate.

\section{References}

Abbate E., Fanucci F., Benvenuti M., Bruni P., Chiari M., Cipriani N., Falorni P., Fazzuoli M., Finocchiaro F., Morelli D., Moretti S., Nebbiai M., Pandeli E., Papini M., Pugliese N, Sagri M., Reale V., Vannucchi P., Venturi F. (2005). Note illustrative della Carta Geologica d' Italia alla scala 1:50.000, Foglio n. 248 (La Spezia). Roma: APAT Dipartimento Difesa del Suolo -Servizio Geologico d' Italia.

Autorità di Bacino Interregionale del Fiume Magra (2006). Piano Stralcio “Assetto Idrogeologico” del Fiume Magra e del Torrente Parmignola. Relazione Generale.

Bini M., Chelli A., Pappalardo M. (2006). Geomorfologia del territorio dell'antica Luni (La Spezia) per la ricostruzione del paesaggio costiero in età romana. Atti Soc. Tosc. Sci. Nat., Mem., Serie A, 111 pp. 57-66.

Convenzione Europea sul Paesaggio (2000). Firenze.

Fara A. (1983). La Spezia, Laterza.

IGM (2005). La città in divenire. Il territorio spezziono dal XIX secolo: immagini e carte. Istituto Geografico Militare, Firenze.

Marino L. (2007). Cave storiche e risorse lapidee: documentazione e restauro. Alinea, Firenze.

Nisio S. (2008). I sinkholes in Liguria. Mem. Descr. Carta Geol. d'It. LXXXV, pp. 399 - 408.

Pintus S. (2004). "Manifestazioni sprugolari nell'ambito del golfo della Spezia“. APAT, in Atti I $^{\circ}$ Seminario- Stato dell'arte sullo studio dei fenomeni di sinkholes e ruolo delle amministrazioni statali e locali nel governo del territorio - Roma 20-21 Maggio pp. 607-614.

Rolla F. - La polla d'acqua dolce di Cadimare nel Golfo della Spezia. http://www.academia.edu/3687712/La_polla_dacqua_dolce_di_Cadimare_nel_Golfo_della_Spezia.

Pascucci M. (2006). La potenza della Povertà. Marx legge Spinoza. Ombre corte Edizioni.

Pizziolo G., Micarelli R. (2003). L'arte delle relazioni. Alinea Editrice. 